\title{
Refractory Granulomatosis with Polyangiitis Presenting as Facial Paralysis and Bilateral Sudden Deafness
}

\author{
Sang Hoon Kim, A Ra Jung, Su II Kim, and Seung Geun Yeo \\ Department of Otorhinolaryngology, School of Medicine, Kyung Hee University, Seoul, Korea
}

Received September 4, 2015

Revised November 9,2015

Accepted December 2, 2015

\author{
Address for correspondence \\ Seung Geun Yeo, MD, PhD \\ Department of Otorhinolaryngology, \\ School of Medicine, \\ Kyung Hee University, \\ 23 Kyungheedae-ro, Dongdaemun-gu, \\ Seoul 02447, Korea \\ Tel $+82-2-958-8474$ \\ Fax $+82-2-958-8470$ \\ E-mailyeo2park@gmail.com
}

Granulomatosis with polyangiitisis [(GPA) or Wegener granulomatosis] is a multi-system disease characterized by granuloma formation and necrotizing vasculitis. GPA classically shows involvement of the respiratory tracts and the renal system. However, locoregional disease is common and may include otologic manifestations. Although otologic involvement can occur during the course of GPA, no report has described facial palsy with sudden sensorineural total deafness with vertigo as the presenting feature of GPA. This case describes a patient with multiorgan involving resistant form of GPA initially presenting with bilateral profound sudden sensorineural hearing loss and left facial paralysis with vertigo. The condition responded well to treatment with rituximab.

J Audiol Otol 2016;20(1):55-58

KEY WORDS: Granulomatosis with polyangiitisis - Bilateral hearing loss · Facial paralysis . Rituximab.

\section{Introduction}

Granulomatosis with polyangiitisis (GPA) is an idiopathic systemic form of vasculitis characterized by involvement of the upper and lower airways and the kidneys. The exact cause of GPA is unknown, but it seems to have characteristics of an autoimmune disease [1].

ENT manifestations are present in the vast majority of patients (73-99\%), and are usually among the first symptoms [2]. Occasionally, ear conditions are the first and only manifestations [3]. Therefore, ENT physicians have a determining role in recognizing the early onset of this disease and starting the proper therapy.

Current first line induction therapy for GPA consists of cyclophosphamide with glucocorticoids, which is successful in most (70-90\%) patients [4]. However, patients resistant to this regimen may be difficult to treat. Although a few patients presenting with otologic symptoms and treated with conven-

This is an Open Access article distributed under the terms of the Creative Commons Attribution Non-Commercial License (http://creativecommons. org/licenses/by-nc/3.0/) which permits unrestricted non-commercial use, distribution, and reproduction in any medium, provided the original work is properly cited. tional therapy, such as cytotoxic drugs and steroids, have been described, this case was a rare and meaningful report in otology to date of a patient presenting with unilateral facial nerve palsy and bilateral profound sudden sensorineural hearing loss with vertigo.

We describe here a patient with a resistant fulminant generalized form of GPA initially presenting as bilateral sudden sensorineural hearing loss and facial palsy. Treatment of this patient with rituximab resulted in significant clinical improvement.

\section{Case Report}

A 47-year-old man visited our hospital due to symptoms of facial paralysis on the left side and abrupt hearing disturbance with dizziness in both ears three days earlier. In physical examination, both tympanic membrane were bulging (Fig. 1). At the time of visit to our hospital, impedance audiometry (Grason-Stadler GSI 33 middle-ear analyzer; Viasys, Conshohocken, PA, USA) was B-type on both sides; and pure tone audiometry (Grason-Stadler GSI 61 clinical; Nicolet Biomedical, Madison, WI, USA) was $95 \mathrm{~dB}$ on the right side and off scale on the left side (Fig. 2A). Tests of auditory brainstem re- 

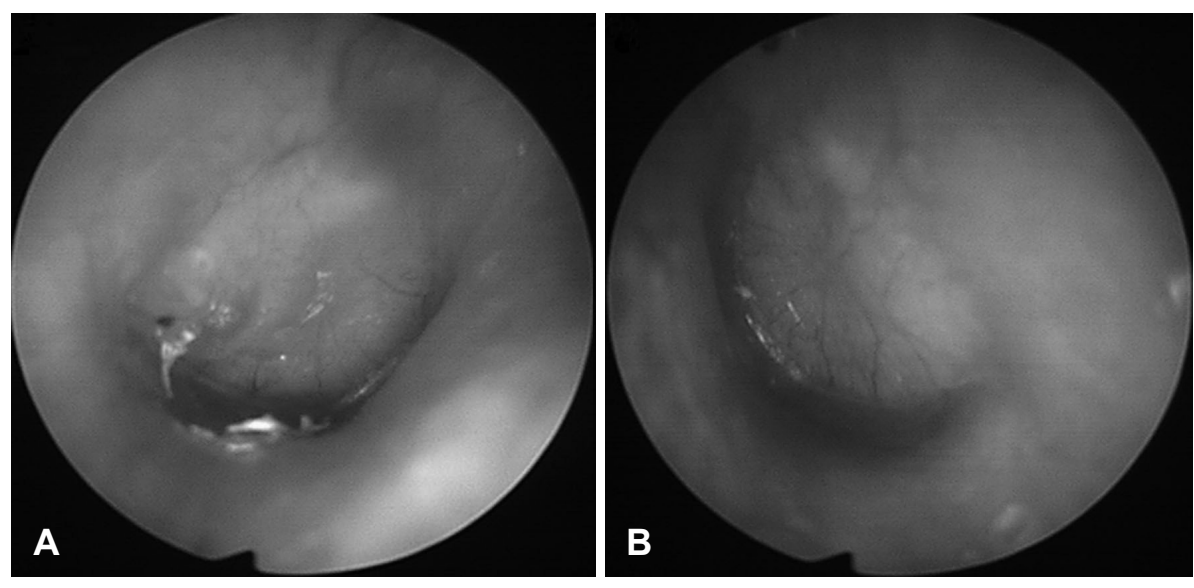

Fig. 1. Right (A) and left (B) tympanic membrane showing bulging.
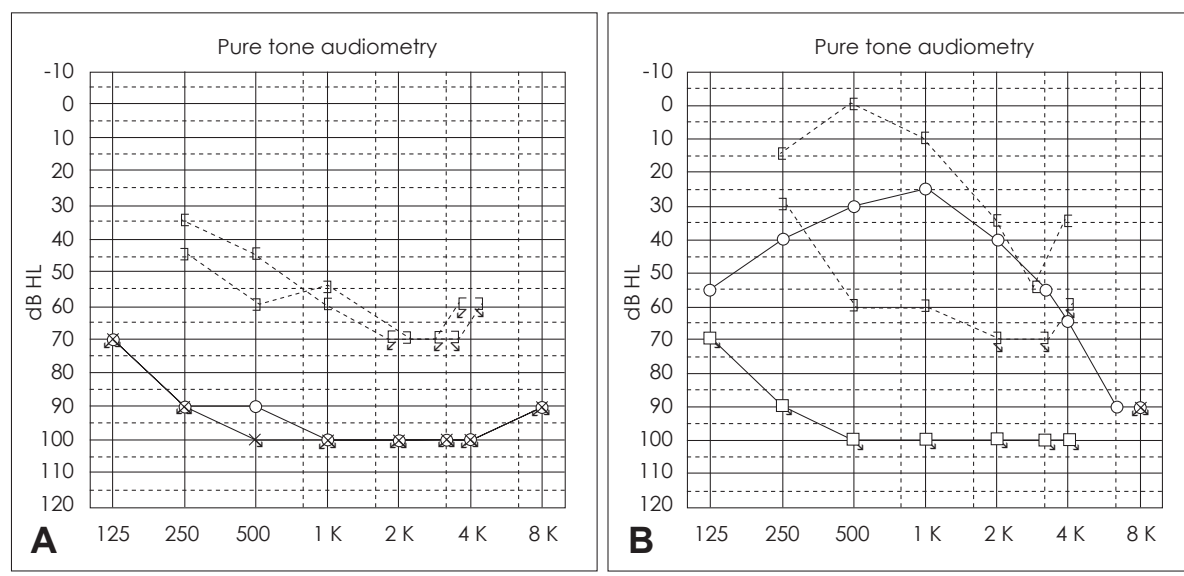

Fig. 2. Pure tone audiometry of the patient at first out patient department visit $(A)$ and after steroid tapering therapy and intratympanic steroid injection (B).
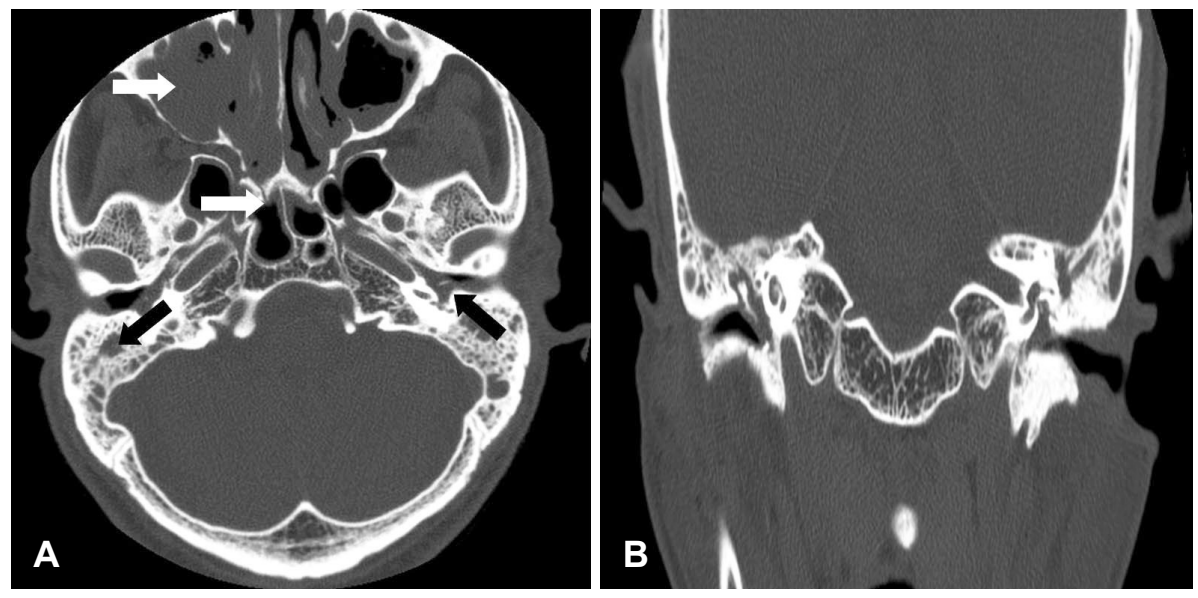

Fig. 3. Axial (A) and coronal (B) views showing fluid density in both middle ear cavities and external auditory canals, with mastoid air cells (black arrows). No abnormality was observed in the facial nerve canal or ossicular chain. Both maxillary and ethmoid sphenoid sinusitis are present (white arrows).

sponse found that the right side formed a V-wave at $80 \mathrm{nHL}$, whereas the left side did not form a V-wave at 90 nHL. Computed tomography scans (General Electric Medical Systems, Milwaukee, WI, USA) showed dense fluid in both external ears and middle ears but no facial canal dehiscence (Fig. 3).

The patient was started on $80 \mathrm{mg} /$ day oral steroids for 12 days. Simple chest X-rays showed multiple nodules in both lung fields, while CT of the chest showed that chest cavity was filled with multiple nodules (Fig. 4A, B). Immunological tests showed significantly higher than normal proteinase- 3 anti-neutrophil cytoplosmic antibodies (ANCA) representing c-ANCA. A definitive diagnosis required a lung biopsy sample. However the patient refused further evaluation.

Following discharge from the hospital, the patient began coughing severely and developed a continuous fever of about $38^{\circ} \mathrm{C}$. In addition, hearing ability again deteriorated. Chest CT 

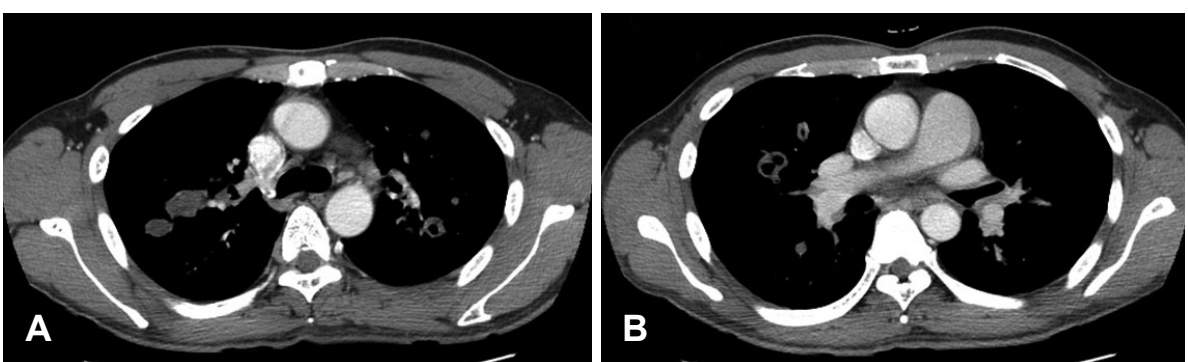

Fig. 4. A, B: Axial views of the patient at admission, showing multiple variable sized cavitary and noncavitary nodules and bronchial wal thickening in both lungs. C, D: Axial views at follow up showing increased size and extent of multiple variable sized cavitary and non-cavitary nodules and bronchial wall thickening with newly developed centrilobular nodules in both lungs.
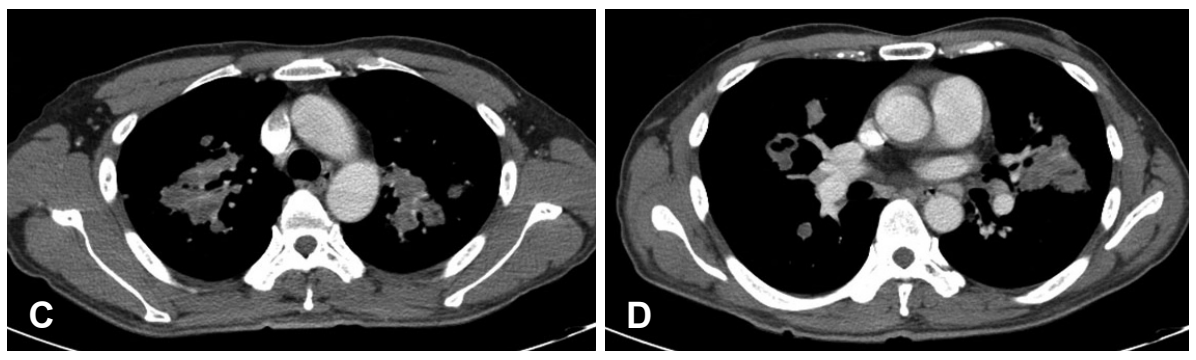

Fig. 5. Lung biopsy showing characteristics of Wegener's granulomatosis. Hematoxylin and eosin staining at (A) $20 \times$ and (B) $40 \times$ original magnification. White arrows indicate a necrotizing granulomatous inflammation with giant cells, palisading histiocytes and nuclear debris. The black arrow indicates hemorrhagic fibrinous exudates fillingthe bronchoalveolar spaces.
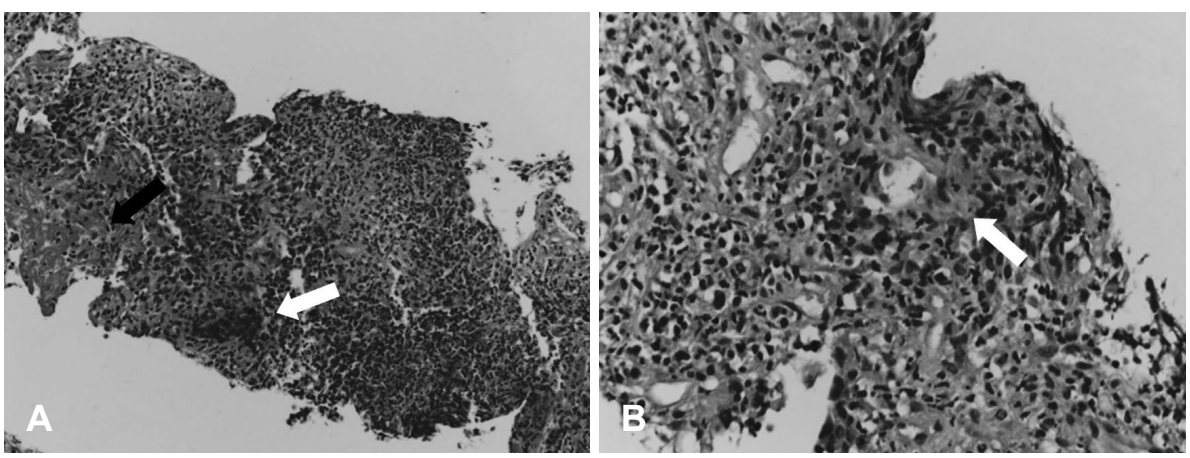

showed increased size and number of nodules in the fine structure of the lungs (Fig. 4C, D). A lung biopsy sample was obtained, and he was started on high-dose intravenous steroids $500 \mathrm{mg} /$ day for three days. After six days, C-reactive protein (CRP) tended to decrease from 18.04 to 4.36, but he showed no improvement in other symptoms including fever.

A lung biopsy taken on day six showed necrotizing granulomatous inflammation with intra alveolar hemorrhagic exudates and capillaritis (Fig. 5), resulting in a diagnosis of GPA. The patient was started on $1000 \mathrm{mg}$ /day cyclophosphamide, along with steroids, two-week interval. However, erythrocyte sedimentation rate, CRP and fever all remained high. He was therefore administered four infusions of rituximab (Mabthera; Roche Pharma, Basel, Switzerland, 500 mg each), an anti-CD20 monoclonal antibody, at two week intervals, along with concurrent IV cyclophosphamide (Endoxan; Bukwang Pharm, Seoul, Korea, $500 \mathrm{mg}$ twice a month for 3 months). After rituximab administration, symptoms including cough, fever, and otologic symptoms, improved significantly. To improve hearing, he received seven intratympanic injections of steroids through the ventilation tubes in each ear. After four months, pure tone audiometry improved on the right side to $32 \mathrm{~dB}$ (Fig. 2B) but did not improve on the left side, and left side facial paralysis improved from $\mathrm{HB}$ grade 5 to $\mathrm{HB}$ grade 2 . Written informed consent was obtained from the patient who participated in this case.

\section{Discussion}

GPA is a relatively rare disease, so treatment focusing on local symptoms in the absence of an accurate diagnosis may have no effect, or even worsen the disease, with the possibility of a secondary infection. Thus, an accurate initial diagnosis and subsequent treatment with steroids and, if necessary, immunosuppressive drugs, are important.

The American College of Rheumatology (ACR) criteria for confirming the diagnosis of GPA was found to have a sensitivity of $88.2 \%$ and a specificity of $92 \%$. A diagnosis of GPA requires at least two of the following four criteria: 1) hematuria (more than 5 red blood cells per visual field or the presence of erythrocyte casts), 2) changes in chest radiographs, 3) ulceration of the mouth and/or nose, and 4) positive histopathological examination. In addition about $80-90 \%$ of these patients are positive for ANCA, which damage vascu- 
lar endothelium and cause its necrosis [5].

In evaluating our patient, we initially focused on the areas usually involved in GPA, including the upper and lower airways and the kidneys. A lung biopsy sample taken from our patient showed necrotizing granulomatous inflammation with intraalveolar hemorrhagic exudates and capillaritis. He also had intranasal inflammation and nodules and cavities in the chest, thereby satisfying more than two of the four ACR criteria.

Ear disorders may be the first and only manifestation of GPA. Otologic involvement may include otitis media with effusion, chronic otitis media, sensorineural hearing loss, vertigo, and/or facial palsy [3]. Proposed mechanisms include cochlear nerve compression by an adjacent granuloma, cochlear immune-complex deposition, and local vasculitis involving cochlear vessels. Progression is generally rapid; however, the condition is occasionally reversible with glucocorticoids or cytotoxic agents [3]. Our patient presented with the otologic symptoms of unilateral facial palsy and bilateral sudden sensorineural hearing loss with vertigo.

Hearing loss was likely due to recurrent serous and/or suppurative otitis media and/or sensorineural hearing loss. Sensorineural and conductive hearing loss occurred with similar frequencies, $47 \%$ and $33 \%$, respectively, but the latter showed a better response to treatment, indicating a poorer prognosis in patients with sensorineural hearing loss [6]. The right side hearing threshold in our patient improved to $32 \mathrm{~dB}$, but left side showed no improvement.

Facial nerve palsy in association with GPA is rare, being present in about $5 \%$ of patients, either alone or in combination with hearing loss; rarely, facial nerve palsy may be the presenting feature. Facial nerve palsy is secondary to compression of the nerve in the middle ear, especially in the presence of a dehiscent facial nerve canal or due to vasculitis [7]. Differential diagnosis is important in patients with facial nerve palsy; other diseases should be considered, such as chronic otitis media and systemic vascular diseases, including sarcoidosis, polyarteritis nodosa and tuberculosis [8].

The standard treatment for systemic GPA is an immunosuppressive drug such as cyclophosphamide to alleviate the disease, along with a glucocorticoid. If these regimens are ineffective, the patient should be treated with rituximab [9], a chimeric monoclonal antibody directed against CD20 that induces B cell apoptosis and depletion in peripheral blood. The importance of ANCA in the pathogenesis of GPA suggests that rituximab reduces ANCA by depleting B cell concentrations. Our patient was initially treated with cyclophosphamide and a glucocorticoid, but symptoms worsened. Addition of rituximab improved symptoms dramatically.

GPA is a rare but dangerous disease, which may progress rapidly if misdiagnosed. This study describes a patient with a rare type of multi-organ involved, treatment resistant, fulminant form of GPA initially presenting as bilateral profound sudden sensorineural hearing loss with vertigo and unilateral facial palsy and illustrates the dramatic effects of rituximab.

\section{Acknowledgments}

This work was supported by the National Research Foundation of Korea (NRF) grant funded by the Korea government (MSIP) (No. 2011-0030072).

\section{Conflicts of interest}

The authors have no financial conflicts of interest.

\section{REFERENCES}

1) Oristrell J, Bejarano G, Jordana R, Monteagudo M, Marí B, Casanovas A, et al. Effectiveness of rituximab in severe Wegener's granulomatosis: report of two cases and review of the literature. Open Respir Med J 2009;3:94-9.

2) Erickson VR, Hwang PH. Wegener's granulomatosis: current trends in diagnosis and management. Curr Opin Otolaryngol Head Neck Surg 2007;15:170-6.

3) Takagi D, Nakamaru Y, Maguchi S, Furuta Y, Fukuda S. Otologic manifestations of Wegener's granulomatosis. Laryngoscope 2002; 112:1684-90.

4) Sánchez-Cano D, Callejas-Rubio JL, Ortego-Centeno N. Effect of rituximab on refractory Wegener granulomatosis with predominant granulomatous disease. J Clin Rheumatol 2008;14:92-3.

5) Wierzbicka M, Puszczewicz M, Bartochowska A, Szyfter W. [The otologic manifestation of Wegener's granulomatosis--review of contemporary achievements in diagnostics and treatment]. Otolaryngol Pol 2012;66:254-8.

6) Bakthavachalam S, Driver MS, Cox C, Spiegel JH, Grundfast KM, Merkel PA. Hearing loss in Wegener's granulomatosis. Otol Neurotol 2004;25:833-7.

7) Ferri E, Armato E, Capuzzo P, Cavaleri S, Ianniello F. Early diagnosis of Wegener's granulomatosis presenting with bilateral facial paralysis and bilateral serous otitis media. Auris Nasus Larynx 2007; 34:379-82.

8) Hern JD, Hollis LJ, Mochloulis G, Montgomery PQ, Tolley NS. Early diagnosis of Wegener's granulomatosis presenting with facial nerve palsy. J Laryngol Otol 1996;110:459-61.

9) Keogh KA, Ytterberg SR, Fervenza FC, Carlson KA, Schroeder DR, Specks U. Rituximab for refractory Wegener's granulomatosis: report of a prospective, open-label pilot trial. Am J Respir Crit Care Med 2006;173:180-7. 